\title{
Radiation Resistant Camera System for Monitoring Deuterium Plasma Discharges in the Large Helical Device*)
}

\author{
Mamoru SHOJI and LHD Experiment Group \\ National Institute for Fusion Science, National Institutes of Natural Sciences, Toki 509-5292, Japan
}

(Received 28 November 2019 / Accepted 5 May 2020)

\begin{abstract}
Radiation resistant camera system was constructed for monitoring deuterium plasma discharges in the Large Helical Device (LHD). This system has contributed to safe operation during two experimental campaigns without serious problems due to radiation (neutrons and gamma-rays). The cameras steadily functioned even in the plasma discharge with the maximum neutron emission rate in FY 2017, though some bright specks temporarily appeared on the images. The cameras have been installed in shield boxes which consist of lead boxes covered with $10 \%$ borated polyethylene blocks in all directions. For optimizing the design of the shield box, the radiation flux distribution was calculated by MCNP-6 code, which reveals the reduction of the radiation flux and the change of the energy spectra in the shield box. Thanks to the optimization, significant extension of the lifetime of the cameras has been realized. Investigation of the influence of the radiation on the CCD image sensor shows that the number of bright specks generally increases with the radiation flux to the camera, which also indicates that some bright specks disappear by the self-annealing process on the image sensor. This phenomenon also highly contributes to the further extension of the lifetime of the radiation resistant cameras.
\end{abstract}

(c) 2020 The Japan Society of Plasma Science and Nuclear Fusion Research

Keywords: radiation resistant camera, neutron, gamma-ray, CCD, deuterium plasma, LHD

DOI: $10.1585 /$ pfr.15.2402039

\section{Introduction}

The first deuterium (D-D) experimental campaign started in March 2017 in the Large Helical Device (LHD), and it continued for the following four months [1]. Neutrons are yielded in deuterium plasmas, and gamma-rays are also collaterally yielded by interactions of neutrons with the peripheral components and the torus hall [2]. Before the D-D experimental campaign, about thirty Charge Coupled Device (CCD) cameras which had been directly mounted at viewports on the vacuum vessel have been used for monitoring LHD plasmas and plasma-wall interactions $[3,4]$. It has been well known that the CCD image sensors are very sensitive to and easily damaged by radiation (neutrons and $\gamma$-rays) [5]. In the D-D experimental campaign, it is predicted that the cameras must be replaced at frequent intervals because of the following two facts. One is that the estimated maximum neutron fluence at the viewports is about $8 \times 10^{9} \mathrm{n} / \mathrm{cm}^{2}$ per one deuterium plasma discharge [6]. The other fact is that the number of damaged CCD image sensors per this neutron fluence is estimated to be about $5 \times 10^{3}$ pixels, which is derived from the experimental data reported in the reference 5. These two facts result in that all the image sensors in standard CCD cameras having $4 \times 10^{5}$ pixels are damaged in about 80 deuterium plasma discharges. This estimation means that all the cameras have to be replaced by two times per one-

author'se-mail: shoji@nifs.ac.jp

*) This article is based on the presentation at the 28th International Toki Conference on Plasma and Fusion Research (ITC28). day plasma discharge operation in LHD, producing large amounts of radioactive wastes and significantly lowers the operating rate. Thus, it was an urgent task to develop a radiation resistant camera system for monitoring deuterium plasma discharges.

In the next section, the specification of the radiation resistant camera system is described. Analyses of a General Monte Carlo N-Particle Transport simulation Code version 6 (MCNP-6) for optimizing the design of the shield box for protecting the cameras from the radiation is presented in section 3 . The successful results of the acquired plasma images at the plasma discharge with the maximum neutron emission yield is depicted in section 4 . In the next section, the investigation of the influence of the radiation on the image sensor is presented, which will provide valuable information for applying the CCD camera in future nuclear fusion reactor plants.

\section{Setup of the Radiation Resistant Camera System}

For protecting the cameras from neutrons and gammarays, five shield boxes were installed on LHD diagnostic stages in the torus hall, as illustrated in Fig. 1. These locations are far from the LHD center by more than $12 \mathrm{~m}$ in order to reduce the influence of high energy neutrons to the cameras. The cameras are covered with a lead $(\mathrm{Pb})$ box (15 mm in thickness) surrounded with $10 \%$ borated polyethylene blocks ( $100 \mathrm{~mm}$ in thickness) in all directions which is the maximum tolerable thickness due the spatial 


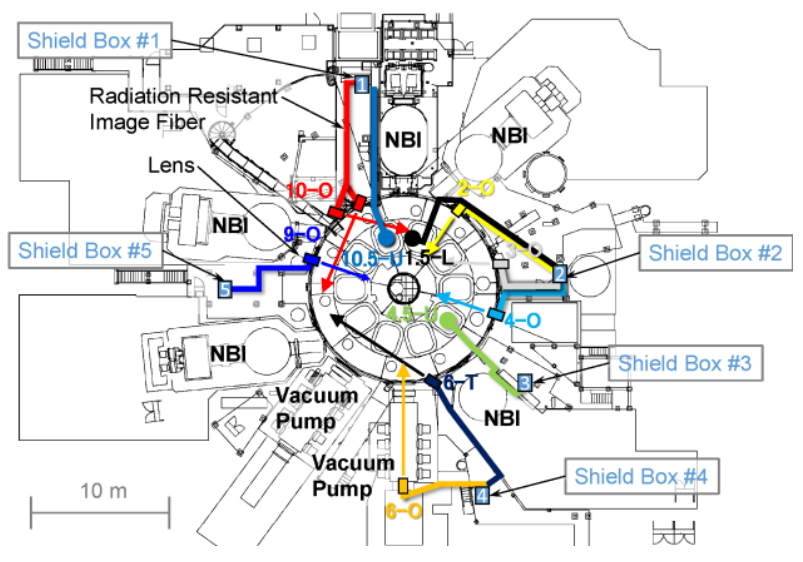

Fig. 1 The top view of the setup of the radiation resistant camera system for monitoring deuterium plasmas in LHD.

limit on the site. The polyethylene blocks are for protecting the cameras from the neutrons, and the lead box is for reducing the gamma-ray flux secondly yielded by the interactions of neutrons with the polyethylene blocks. Two bended slit halls on the up and bottom sides are opened for air ventilation and as ducts for power supply cables, image fibers, signal lines and optical fibers, etc. The lead box includes optics for connecting the image fibers to the CCD cameras (Toshiba IK-CU44, IK-C44 H, IK-M44 H, Hitachi KP-D20A, and KP-D20B), camera controllers (Toshiba IK-UM44 H), media converters (Nanahoshi Kagaku VP205SC), and a serial device server (Moxa NPort5410) for controlling the cameras.

Eight optical lenses were mounted at the viewports on the vacuum vessel in the first deuterium experimental campaign in FY 2017. In the next experimental campaign in FY 2018, three optical lenses were additionally mounted. Plasma images at the viewports are transferred to the cameras via the radiation resistant image fibers (with 30,000 pixels and approximately $10-20 \mathrm{~m}$ in length). The video output signal (NTSC) from the camera controllers are transferred to the control room via the media converters through the optical fibers. The signal is captured by a video encoder and stored in RAID disks or a data acquisition (DAQ) system for LHD.

\section{Optimization of the Design of the Shield Box}

The General Monte Carlo N-Particle Transport simulation Code version 6 (MCNP-6) [7] was applied for optimizing the structure of the shield box, in which an LHD model for one helical coil pitch angle (36 degrees in the toroidal direction) is included with a cylindrical torus hall model for calculating the radiation flux distribution in the hall [6]. Figure 2 illustrates the side and top cross-sectional view of the three-dimensional model of the shield box which consists of a lead box and $10 \%$ borated polyethylene blocks with the slit halls. In this model, the helical

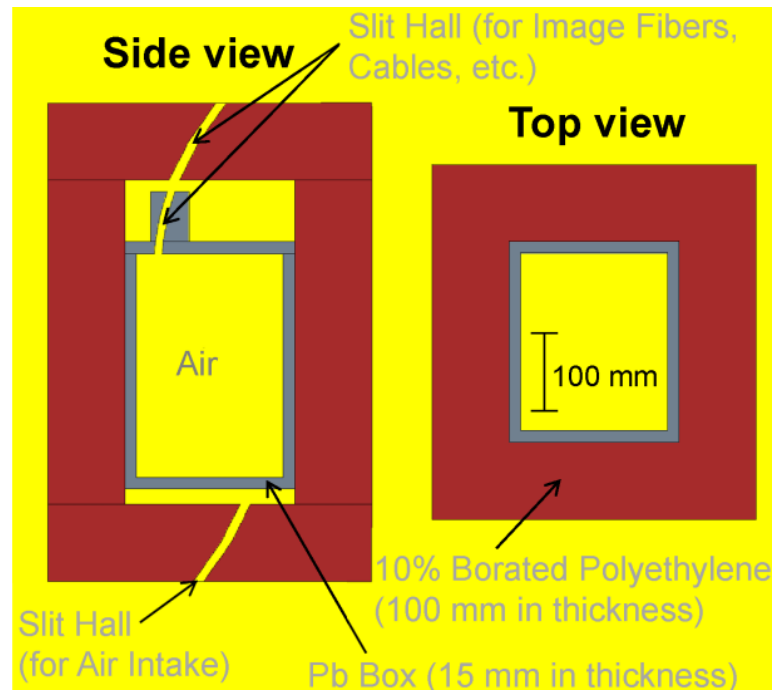

Fig. 2 The side and top cross-sectional view of the threedimensional model of the shield box for the MCNP code.

symmetry of the plasma and the peripheral components is assumed. Neutrons with an energy of $2.45 \mathrm{MeV}$ by the bulk deuterium-deuterium reaction are yielded from circular shaped torus plasma locating at the center of the vacuum vessel.

Figures 3 (a) and (b) give the simulation result of the vertical cross-section of the neutron and gamma-ray flux distribution in the torus hall at a toroidal angle crossing the shield box \#2 for a neutron emission rate of $1.0 \mathrm{n} / \mathrm{s}$, respectively. This shield box is chosen as a representative one for the simulation because of no significant toroidal variation of neutron flux in the torus hall around LHD [8]. Figure 3 (a) shows that the neutron flux is reduced at the position of the shield box by approximately one-order of magnitude compared to that in the vacuum vessel. On the other hand, as illustrated in Fig. 3 (b), while the gamma-ray flux decreases by about one-order of magnitude around the shield box, the $10 \%$ borated polyethylene blocks enhance the gamma-ray flux at the LHD side in the shield box. This simulation result indicates that lead box is essential for protecting the cameras from the enhanced gamma-rays.

The side and top cross-sectional view of the neutron and gamma-ray flux distribution around the shield box \#2 are presented in Figs. 4(a) and (b). These simulations show that the neutron and gamma-ray flux in the lead box is reduced by a factor of about 30 and 10 compared to those around the shield box (LHD side), respectively. Figures 5 (a) and (b) present the calculated neutron and gamma-ray spectra at the shield box, respectively. The solid black lines represent the background spectra which is calculated for no shield box. The colored dotted lines are the calculated spectra at the inside of the lead box, showing that the neutron flux is attenuated in the wide neutron energy range by a factor of about 40 compared to the background flux. The gamma-ray flux in the high energy 


\section{(a) Neutron flux distribution}

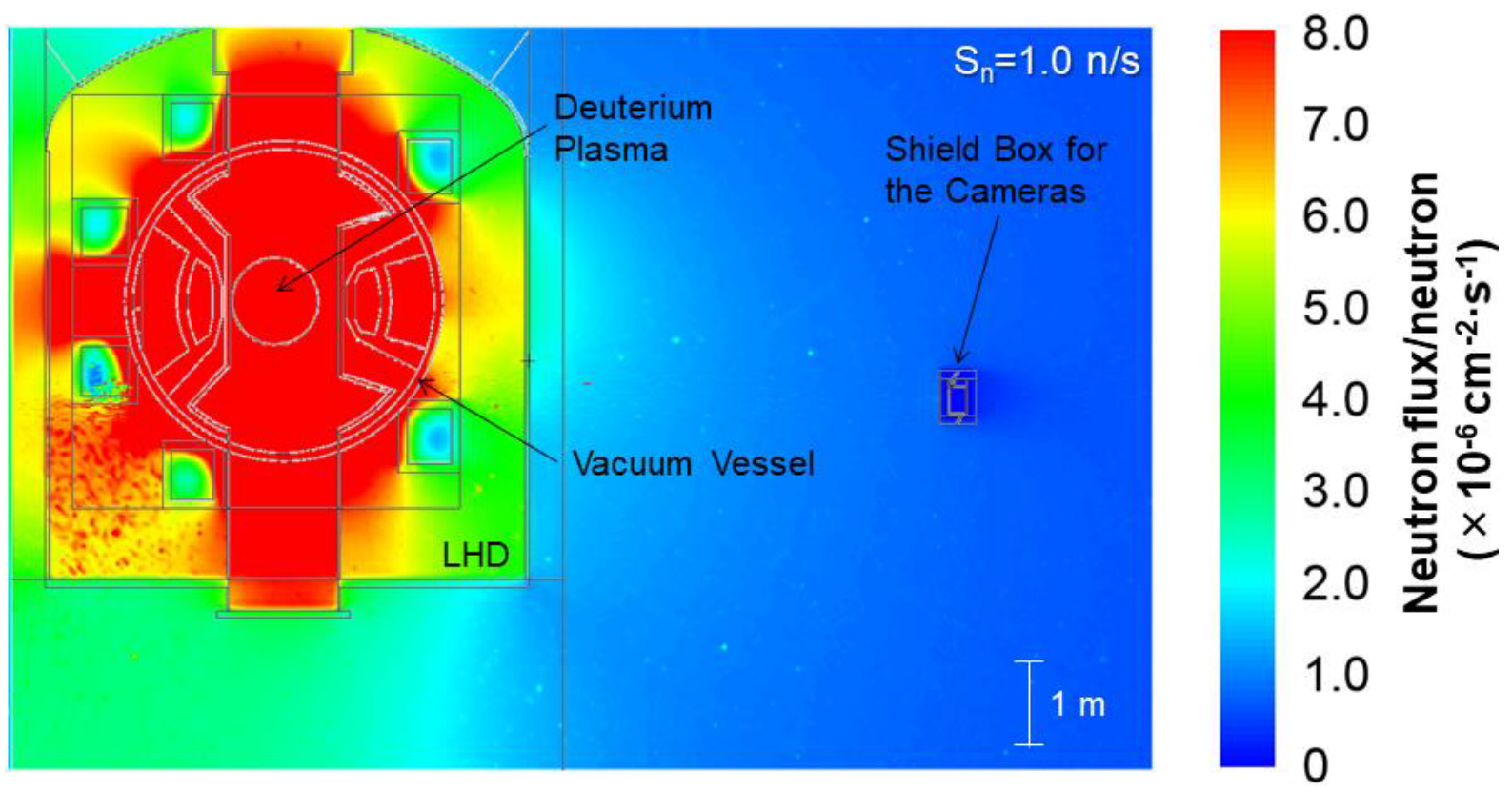

\section{(b) $\quad \gamma$-ray flux distribution}
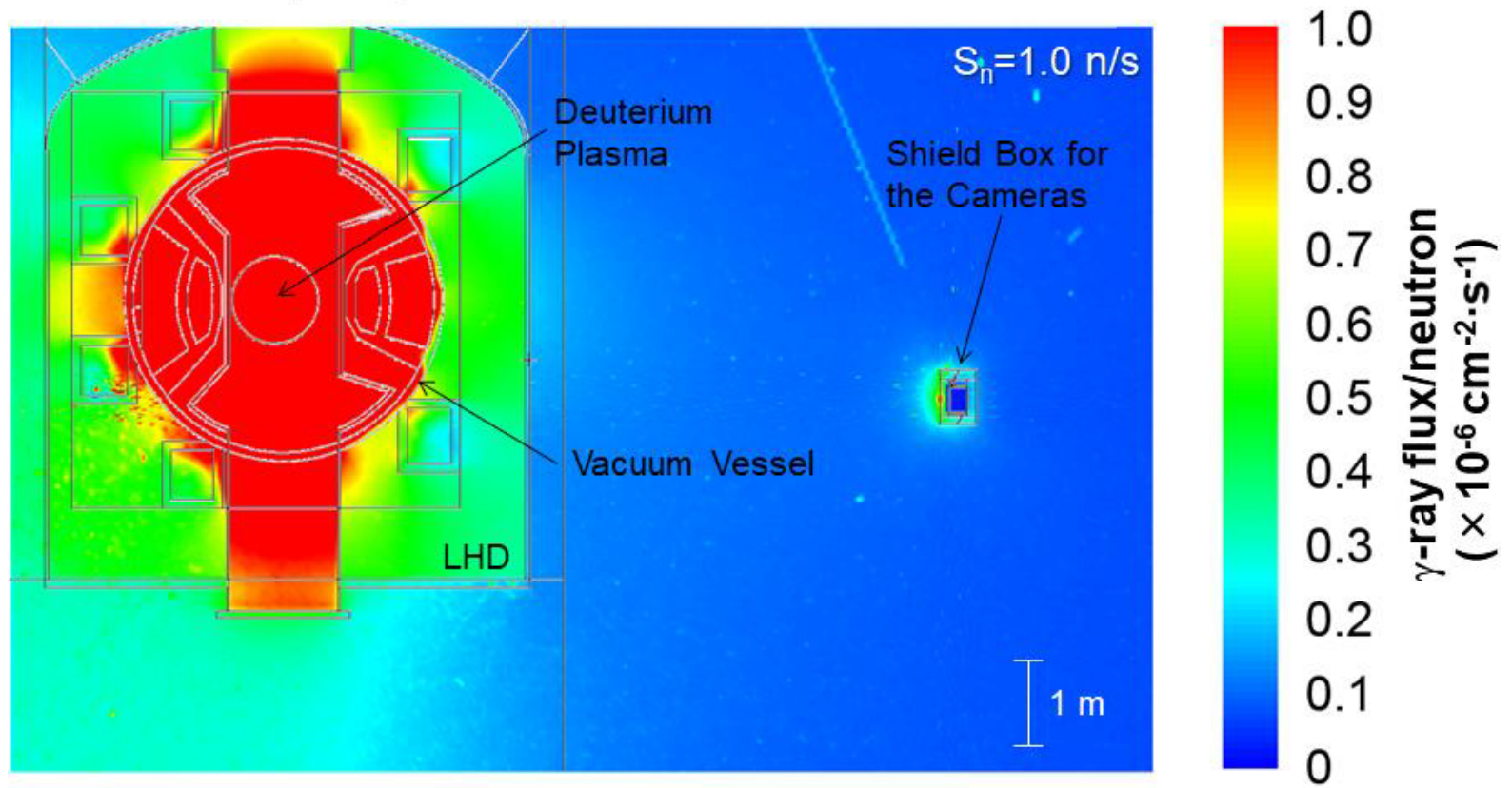

Fig. 3 The simulation of the vertical cross-section of (a) the neutron and (b) the gamma-ray flux distribution in the torus hall for a neutron emission rate of $1.0 \mathrm{n} / \mathrm{s}$ in the LHD deuterium plasma.

range $(>0.4 \mathrm{MeV})$ is reduced by a factor of about 2 or 3 , and the flux in the low energy range $(<0.4 \mathrm{MeV})$ is lowered by about one order of magnitude compared to the back ground flux (solid black line). The shielding performance of the lead box for the gamma-rays is investigated by changing the thickness of the lead plates (components of the lead box). Colored broken lines in Figs. 5 (a) and (b) give the calculated neutron and gamma-ray spectra in the shield box \#2 in the case where the thickness of the lead plates is changed from 5 to $25 \mathrm{~mm}$. The calculations show that the thicker lead plates are effective for reducing gamma-ray flux in the high energy range. The increment of the reduction ratio for $25 \mathrm{~mm}$ is about $20 \%$ compared to that for $15 \mathrm{~mm}$. Lead plate is a heavy and toxic material which has to be handled with care. Considering this complicatedness, the thickness of the lead plate adopted in the 


\section{(a)}

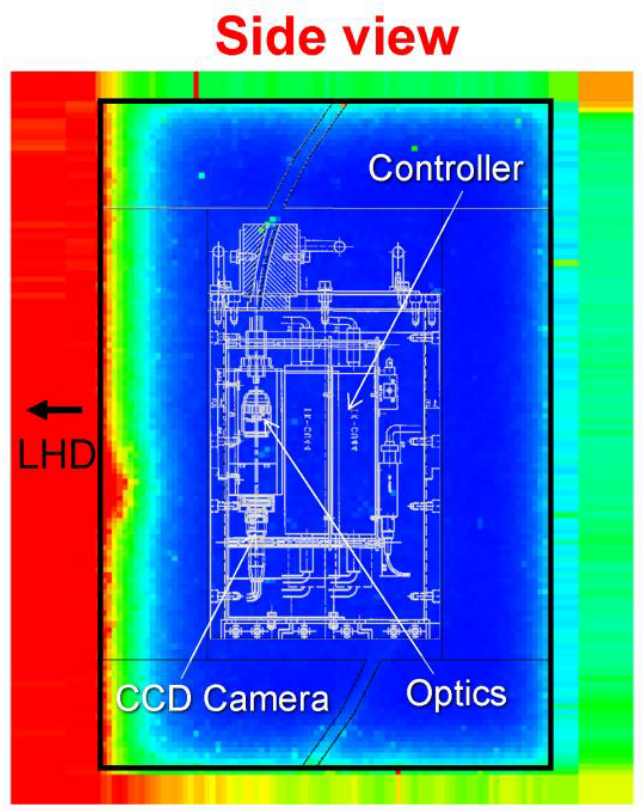

Neutron flux distribution

Top view

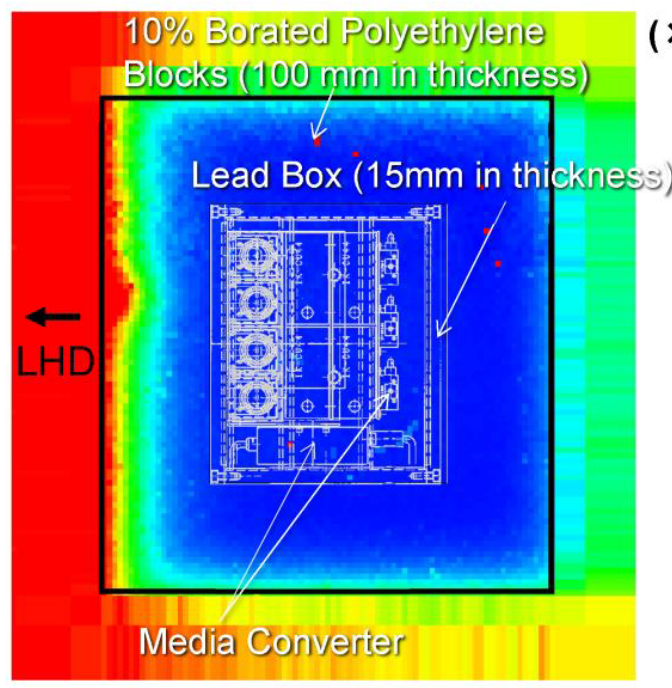

In the lead box $1.6 \times 10^{-8} \mathrm{~cm}^{-2} \cdot \mathrm{s}^{-1}$

$$
\mathrm{S}_{\mathrm{n}}=1.0 \mathrm{n} / \mathrm{s}
$$

Neutron flux Ineutron $\left.\times 10^{-7} \mathrm{~cm}^{-2} \cdot \mathrm{s}^{-1}\right)$

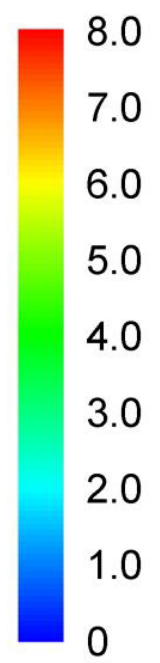

6.0

5.0

4.0

3.0

2.0

0

\section{(b)}

\section{$\gamma$-ray flux distribution}

Side view

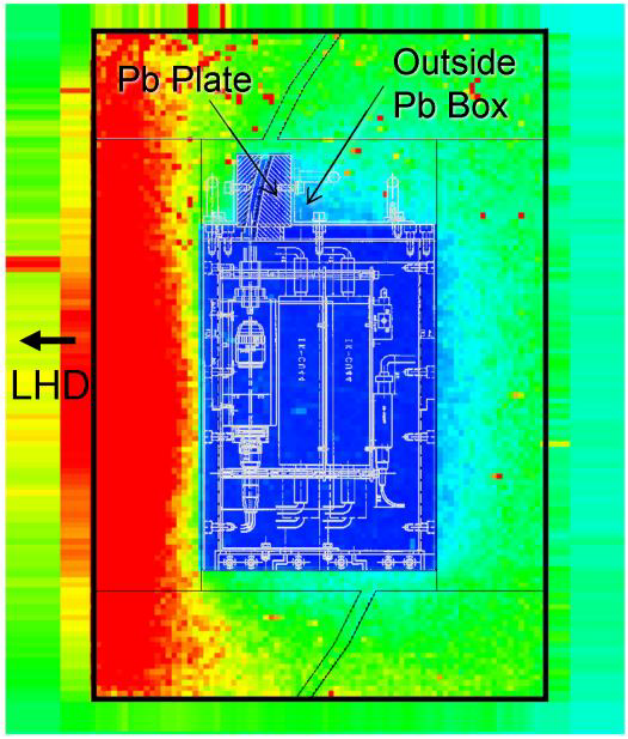

\section{Top view}

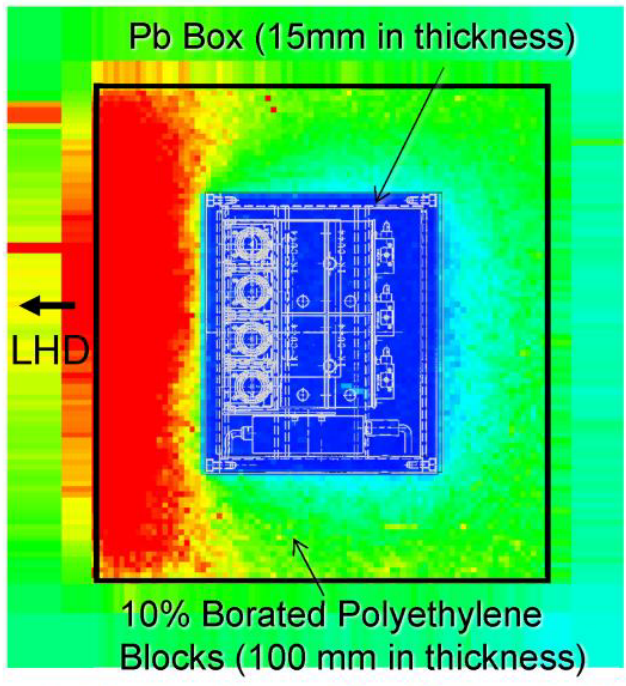

In the lead box 1.9 $\times 10^{-8} \mathrm{~cm}^{-2} \cdot \mathrm{s}^{-1}$
$\mathrm{S}_{\mathrm{n}}=1.0 \mathrm{n} / \mathrm{s}$

$\gamma$-ray flux

Ineutron

$\left(\times 10^{-7} \mathrm{~cm}^{-2} \cdot \mathrm{s}^{-1}\right)$

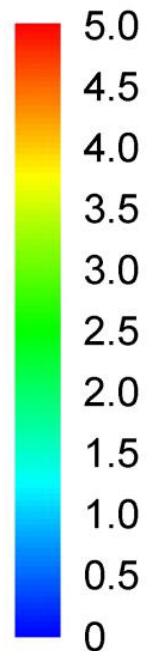

Fig. 4 The simulation of the side and top cross-section of (a) the neutron and (b) the gamma-ray flux distribution in the shield box \#2.

shield box $(15 \mathrm{~mm})$ seems to be reasonable.

The neutron and gamma-ray spectra at a space between polyethylene blocks and the lead box (indicated in Fig. 2) are shown as a blue broken line in Figs. 5 (a) and (b), respectively. The gamma-ray flux in the low energy range is higher than that of the background flux (solid black line). This is because the gamma-rays are yielded in the polyethylene blocks in the LHD side as shown in Fig. 4 (b). On the other hand, the flux in the high energy range is lower than the background flux by more than a factor of two, although the flux is higher than that inside of the lead box with the thickness of $15 \sim 25 \mathrm{~mm}$. This simulation result suggests that the space between the polyethylene blocks and the lead box, which is located behind lead plates from the LHD side, can be effectively used for a space for protecting devices from high energy gamma-rays (not from low energy rays). 
(a)

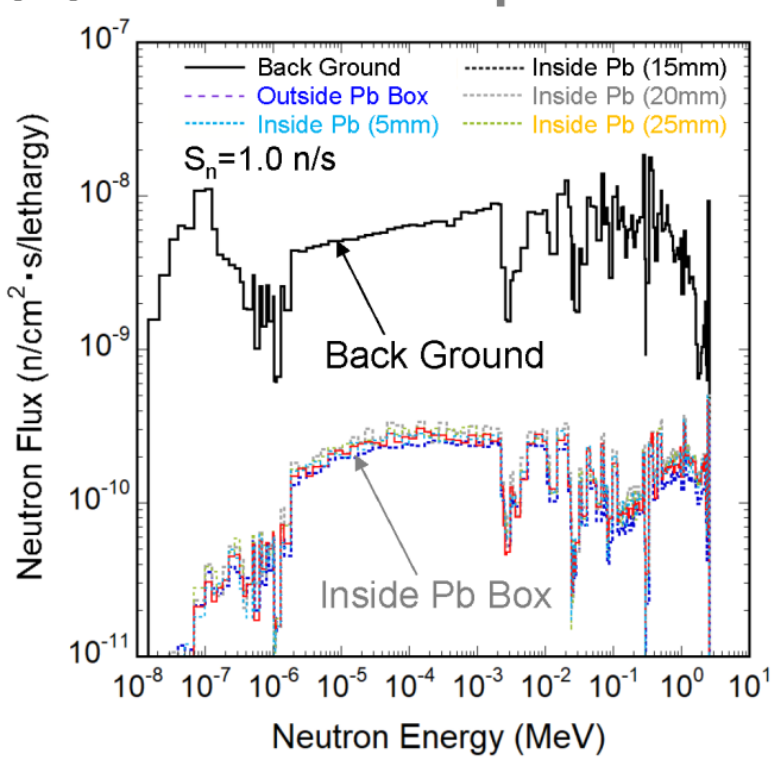

(b) $\gamma$-ray Spectra

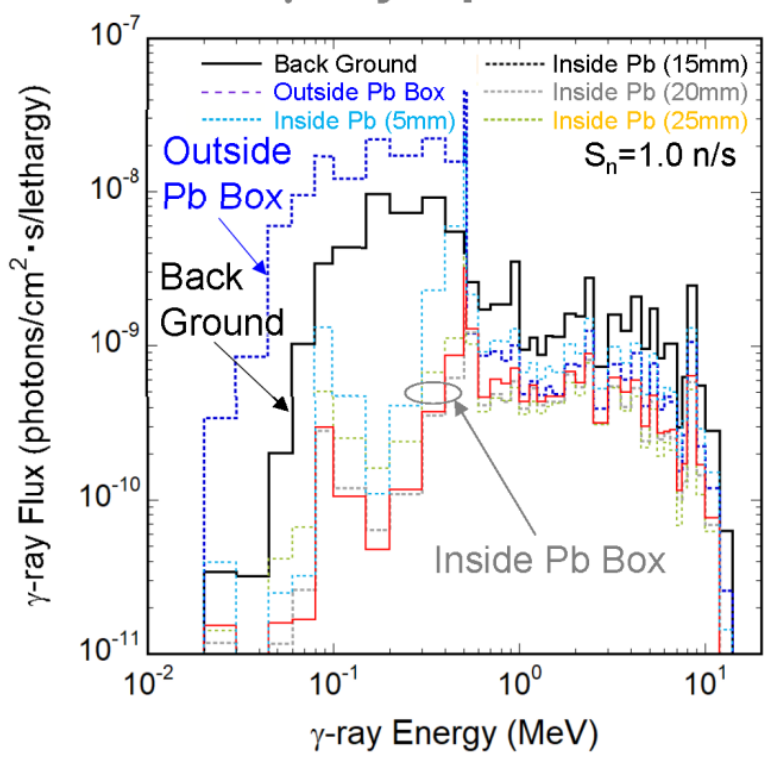

Fig. 5 (a) The calculated neutron spectra at the shield box, and (b) the calculated gamma-ray spectra at the shield box. The solid black lines represent the background spectra. The colored dotted lines present the spectra in the lead box with various thicknesses of the lead plates. The blue broken lines indicates the spectra at a space between polyethylene blocks and the lead box.

\section{Images in the Deuterium Plasma Discharge with the Maximum Neutron Emission Rate}

In the deuterium plasma discharges in the experimental campaign in FY 2017, the maximum neutron emission rate of $3.3 \times 10^{15}(\mathrm{n} / \mathrm{s})$ was achieved. Plasma images taken with the radiation resistant camera systems at the maximum neutron emission rate are shown in Fig. 6. The positions of the optical lenses for the cameras are illustrated in Fig. 1. From the simulation results by the MCNP-6 code in Fig. 4, the neutron and gamma-ray fluxes inside of the lead box are calculated to be $5.3 \times 10^{-7}$ and $6.3 \times 10^{-7} \mathrm{~cm}^{-2} \cdot \mathrm{s}^{-1}$, respectively. It was found that the cameras operate without any problems even in the plasma discharge with the maximum neutron emission rate. Some small bright specks, which are indicated by arrows, appeared in the images as shown in Fig. 6. While these specks disappeared in the next frame, new bright specks emerged at different positions. These temporal bright specks have not disturbed the monitoring of the plasma, proving the durability of the radiation resistant camera systems in the deuterium plasma discharges.

\section{Investigation of the Influence of the Cumulative Radiation Flux on the Image Sensor}

It was believed that the bright specks on CCD image sensors permanently remain after the exposure to radiation flux. A positive correlation between the number of the specks and the cumulative exposed neutron flux was reported [5]. The background images which are taken before the ignition of plasma discharges are a good indicator to check the influence of the radiation on the image sensor. The change of the background image as the increase of the cumulative radiation flux was investigated. The absolute neutron emission yield has been routinely monitored with a neutron diagnostic system [9]. The radiation flux exposed to the camera system is derived by multiplying the simulation result (Fig. 4) by the observed emission yield. Figures 7 (a) and (b) present the change of the background images as the cumulative radiation fluxes in the two experimental campaigns (FY 2017 and FY 2018), respectively. The images taken with the camera (Hitachi KPD20B) mounted at the tangential port (6-T) are shown as a representative. For highlighting the bright specks, brightness and contrast of the images are enhanced. The figures clearly show that the number of specks generally increases with the cumulative radiation flux. The parameters $Y_{\mathrm{n}}$ and $Y_{\gamma}$ are the cumulative neutron and gamma-ray fluxes exposed to the cameras in each experimental campaign, respectively. The camera was turned off at the end of the experimental campaign in FY2017, and it was turned on before the next campaign in FY 2018. The number of specks at the beginning in FY 2018 (left figure in Fig. 7(b)) is lower than that at the end of the previous campaign (right figure in Fig. 7 (a)). For example, one of the specks appearing in FY 2017 (indicated by a blue arrow) disappeared at the beginning in FY2018. This phenomenon can be explained by the so called self-annealing which was reported in the CCD camera mounted in the JET tokamak [10]. In LHD, this self-annealing is found in some pixels even in 

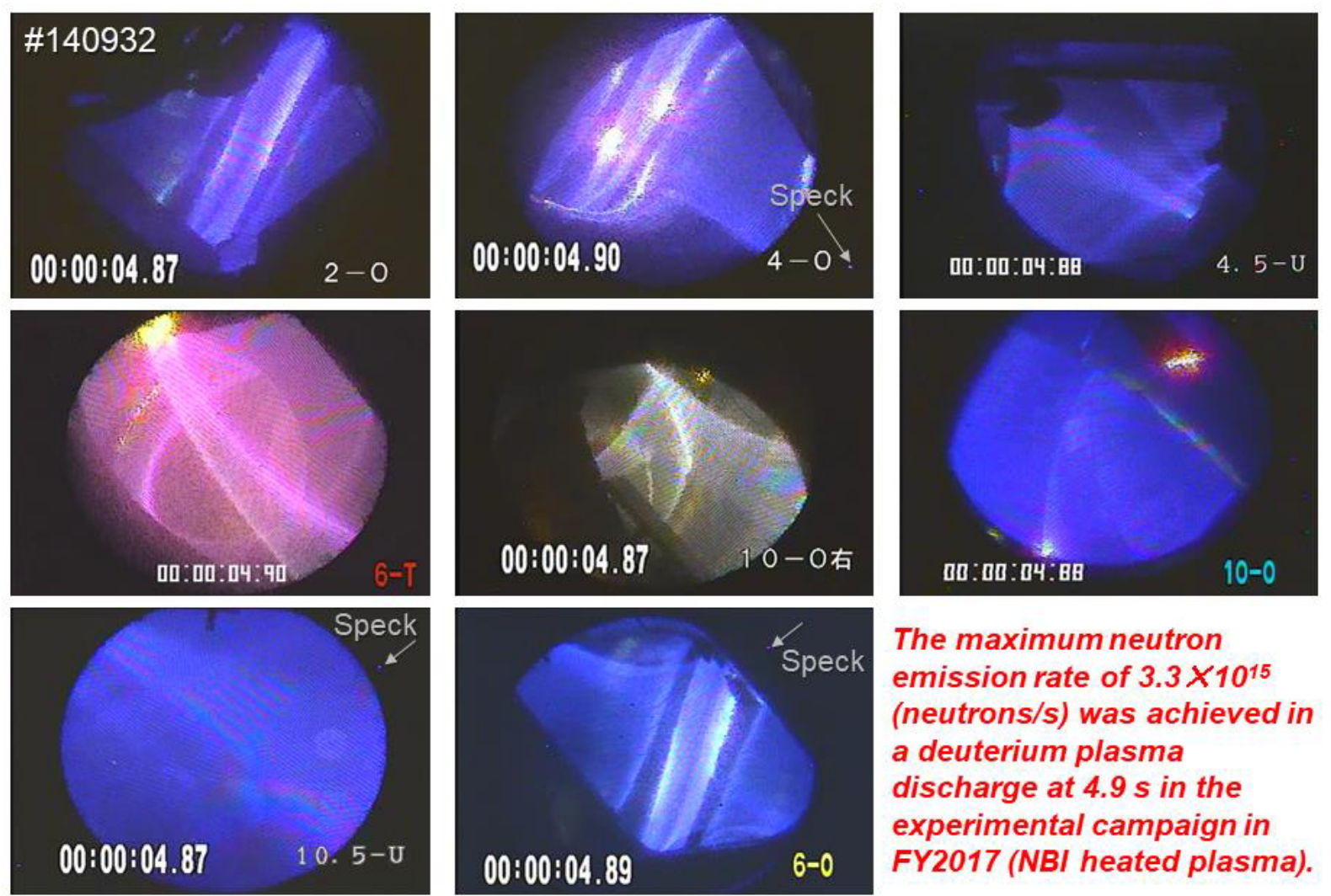

The maximum neutron emission rate of $3.3 \times 10^{15}$ (neutrons/s) was achieved in a deuterium plasma discharge at $4.9 \mathrm{~s}$ in the experimental campaign in FY2017 (NBI heated plasma).

Fig. 6 Plasma images taken with the radiation resistant camera systems at the time of the maximum neutron emission rate in a deuterium plasma discharge in FY 2017.

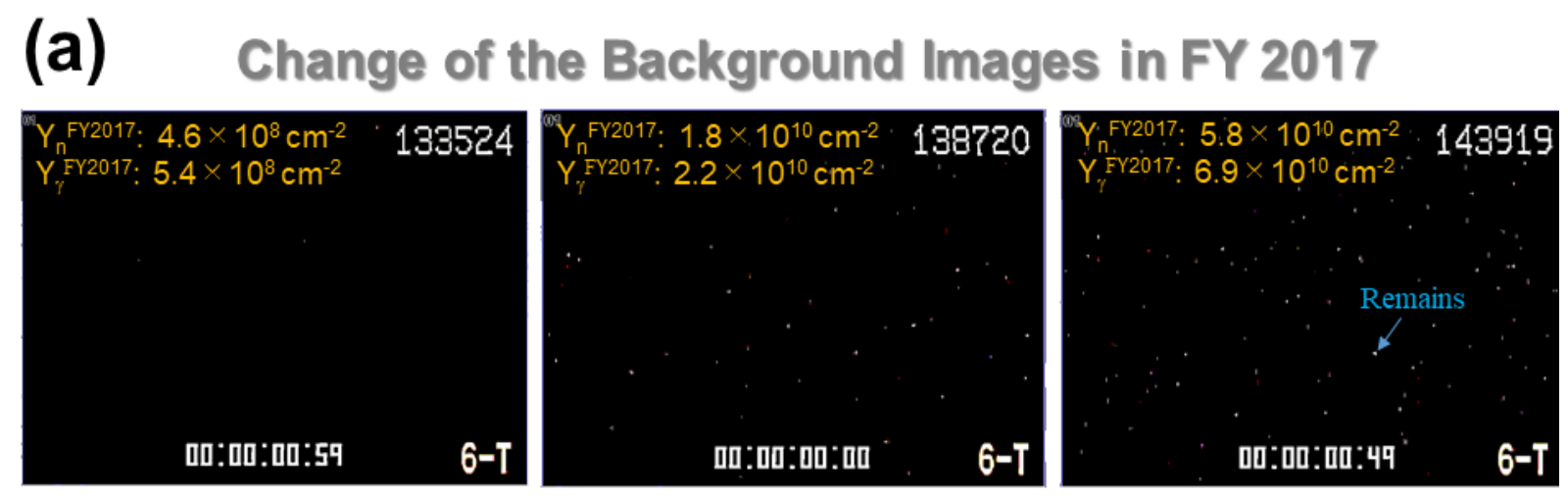

\section{(b) Change of the Background Images in FY 2018}

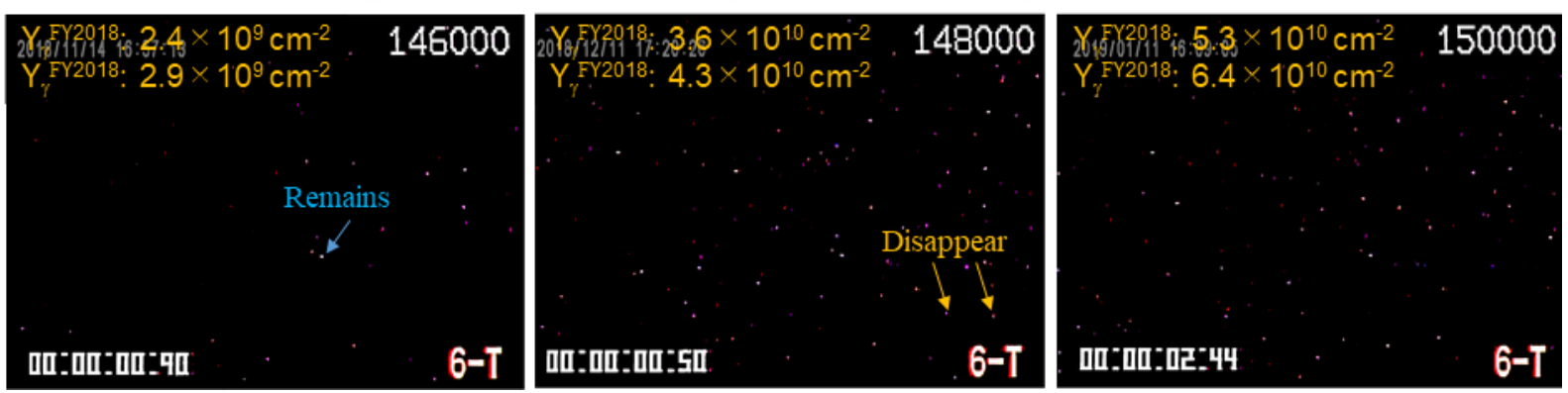

Fig. 7 The change of the background images taken before the ignition of the plasma discharges as the cumulative radiation fluxes in the two experimental campaigns (FY 2017 and FY 2018), respectively. 
one experimental campaign as shown by an orange arrow in Fig. (b). The self-annealing has highly contributed to the extension of the lifetime of the camera systems.

\section{Summary}

Radiation resistant camera systems for monitoring deuterium plasma discharges have been successfully constructed. Investigation using the MCNP-6 code reveals that the neutron and gamma-ray fluxes in the lead box are reduced by a factor of about 30 and 10 compared to those around the shield box, respectively. The calculations of the energy spectra show that the neutron flux decreases in all energy ranges by more than one-order of magnitude, and the gamma-ray flux in the high energy range is reduced by a factor of two or three although the flux in the low energy range is significantly lowered by about one-order of magnitude. The simulation indicates that the thickness $(15 \mathrm{~mm})$ of the lead plates is reasonable because the thicker plates $(25 \mathrm{~mm})$ lower the gamma-ray flux in the high energy range only by about $20 \%$. The radiation resistant cameras have succeeded in operating without serious problems in the deuterium plasma discharge with the maximum neutron emission rate, though some temporary bright specks appeared on the images. The investigation of the change of the background images in the two experimental campaigns in FY 2017 and 2018 shows that the number of specks generally increases with the cumulative radiation flux, and indicates that some specks disappear by self-annealing. This phenomenon has highly contributed to the significant extension of the lifetime of the cameras in deuterium experimental campaigns in LHD.

\section{Acknowledgments}

This work is performed under the auspices of the NIFS budgets (UFEX301 and ULPP801). The author (M.S.) would like to thank Dr. K. Ogawa and Prof. T. Nishitani for the use of the computational resource for the MCNP code. He is also grateful for the support for computing the neutron and gamma-ray flux in the shield box using the MCNP code, and expresses special thanks to the NIFS technical staff for their support for the installation of the radiation resistant camera system and the shield box in the LHD torus hall.

[1] Y. Takeiri et al., IEEE Trans. Plasma Sci. 46, 2348 (2018).

[2] T. Nishitani et al., Plasma Fusion Res. 14, 3405048 (2019).

[3] M. Shoji et al., Nucl. Fusion 55, 053014 (2015).

[4] M. Shoji et al., J. Plasma Fusion Res. SERIES 3, 440 (2000).

[5] Y. Tanimura et al., J. Nucl. Mater. 258-263, 1812 (1998).

[6] T. Nishitani et al., Plasma Fusion Res. 11, 2405057 (2016).

[7] D.B. Pelowits (Ed.), MCNP6 users Manual, LA-CP-1300634, Los Alamos National Laboratory (2013).

[8] T. Nishitani et al., Progress in Nuclear Science and Technology 6, 48 (2019).

[9] M. Isobe et al., IEEE Trans. Plasma Sci. 46, 2050 (2018).

[10] A. Huber et al., Fusion Eng. Des. 123, 669 (2017). 